\title{
Rezension: PraxisHandbuch Gleichbehandlung
}

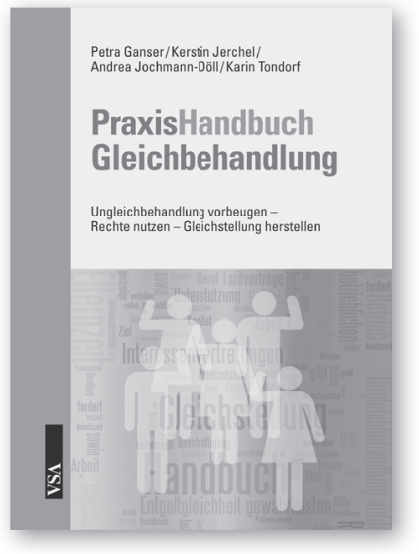

ver.di-Vereinte Dienstleistungsgewerkschaft (Hrsg.) Petra Ganser, Kerstin Jerchel, Dr. Andrea Jochmann-Döll, Dr. Karin Tondorf PraxisHandbuch Gleichbehandlung - Ungleichbehandlung vorbeugen - Rechte nutzen Gleichstellung herstellen 415 Seiten, Erscheinungsjahr 2011, ISBN 978-3-89965-435-0 VSA: Verlag, Hamburg

Das von der Dienstleistungsgewerkschaft ver.di herausgegebene „PraxisHandbuch Gleichbehandlung“ richtet sich an Tarifvertragsparteien, die betrieblichen Interessenvertretungen, aber auch an Personalverantwortliche und Betroffene und liefert diesen einen Handlungsleitfaden zur Unterbindung von Ungleichbehandlung von Frauen und Männern in der Arbeitswelt.

Geschrieben wurde das Handbuch von Petra Ganser und Kerstin Jerchel von der ver.di Bundesverwaltung sowie von den Wissenschaftlerinnen Dr. Andrea Jochmann-Döll und Dr. Karin Tondorf, wobei letztere ebenfalls Mitglied der Kommission Arbeits-, Gleichstellungs- und Wirtschaftsrecht des djb ist.

Das Handbuch besteht aus fünf Teilen, die sich mit der Gleichbehandlung von Frauen in den Bereichen Arbeitsentgelt, Arbeitszeit, Beschäftigung und mit möglichen Präventivmaßnahmen sowie der rechtlichen und gerichtlichen Durchsetzung beschäftigen. Im Anhang werden außerdem die wichtigsten einschlägigen Rechtsvorschriften und Gesetze noch einmal übersichtlich abgedruckt.

Jedes Kapitel beginnt mit der Darlegung von Zahlen und Fakten sowie der gesetzlichen Regelung und Rechtsprechung auf deutscher sowie europäischer Ebene zum jeweiligen Thema. Ferner wird in jedem Kapitel aufgezeigt, wie in den verschiedenen Bereichen Ungleichbehandlung konkret erkannt und Gleichbehandlung hergestellt werden kann. Abschließend werden stets hilfreiche Links, Literaturhinweise und einschlägige Gerichtsur- teile aufgeführt, so dass die Leserin und der Leser das durch das Handbuch erworbene Wissen noch vertiefen kann.

Im umfangreichsten ersten Teil, der sich mit der Gleichbehandlung bei den verschiedenen Bestandteilen des Arbeitsentgelts befasst, wird außerdem sehr ausführlich und anwenderfreundlich das unter Förderung der Hans-Böckler-Stiftung von den beiden Autorinnen Dr. Andrea Jochmann und Dr. Karin Tondorf entwickelte Prüfungskonzept eg-check vorgestellt. Mit diesem Instrument kann Entgeltungleichheit nicht nur bei gleicher, sondern auch bei gleichwertiger Arbeit aufgedeckt werden.

Auch in den Teilen zwei und drei, welche die Gleichbehandlung bei der Arbeitszeit und Arbeitsunterbrechung sowie bei der Beschäftigung behandeln, werden Prüfungsinstrumente aufgezeigt, mit denen die Benachteiligung von Frauen festzustellen ist.

Besonders positiv hervorzuheben ist, dass der Titel hält, was er verspricht, denn es handelt sich tatsächlich um ein praxistaugliches Handbuch.

Zum einen schaffen es nämlich die Autorinnen, durch übersichtliche Abbildungen und anhand von Praxisbeispielen das schwierige Thema auch für den juristischen Laien gut verständlich aufzubereiten.

Zum anderen werden den Tarifvertragsparteien, betrieblichen Interessenvertretungen, Gleichstellungsbeauftragten und Personalverantwortlichen konkrete Prüfungsinstrumente, Checklisten sowie Formulierungsbeispiele an die Hand gegeben, um Gleichstellung auf tariflicher und betrieblicher Ebene tatsächlich herzustellen.

Abgerundet wird das Werk mit den beiden letzten Teilen, in denen die Rechte und Pflichten der betrieblichen Parteien dargelegt und diesen sowie den Betroffenen konkrete Durchsetzungsstrategien aufgezeigt werden.

Insgesamt handelt es sich somit um ein informatives und anwenderfreundliches Buch, welches der Leserin und dem Leser eine wirkliche Unterstützung zur Feststellung von Ungleichbehandlung und Herstellung von Gleichstellung bietet. (Sonja Prothmann, Mitglied der Kommission Arbeits-, Gleichstellungsund Wirtschaftsrecht des djb; Rechtsanwältin, Frankfurt am Main)

\section{Feministischer Juristinnentag}

\section{6. bis 8. Mai 2011 in Frankfurt am Main}

\author{
Alexandra Evalis Hornung \\ Mitarbeiterin des djb-Projekts „Aktionärinnen fordern Gleichbe- \\ rechtigung“; Rechtsanwältin, Berlin
}

\footnotetext{
Warum heute noch Frauenthemen? Warum heute noch Feminismus?

- schallt es aus dem Medienwald heraus. Falsche Frage, wie sich beim 37. Feministischen Juristinnentag (FJT) herausstellt.
}

Vielmehr müsste es heißen: Warum sind Frauenthemen noch immer Nischenthemen, Feministinnen tabu? Der FJT kann zwar dies nicht beantworten, ist dieser Tage aber eines der wenigen Fenster zur präzisen Betrachtung des Status quo der Gleichstellung in Deutschland.

Nicht nur die AGs („Eheverträge nach der Unterhaltsrechtsreform“, „Opferentschädigungsgesetz“, „Mittelbare Diskriminierung im Einkommenssteuerrecht“, „Anforderungen mittel- 
barer und mehrdimensionaler Diskriminierung an Gesetzgebung und Rechtsdurchsetzung“, „Lebensplanung und Unterhaltsrecht“, „Gerichtspsychologische Gutachten“, „Bemessung der Regelsätze aus frauenpolitischer Sicht“, „Täterinnen“ und „Intersektionalität“) verheißen ein spannendes Tagungswochenende. Beim Workshop „Zur Geschichte und Struktur des FJT" für Neueinsteigerinnen am Freitag heißt es plötzlich: „Scheut euch nicht, die Älteren anzusprechen“. Bitte was? Ginge es nach der Familienministerin, denke ich, hätten die doch gar nichts mehr zu sagen.

Zum Auftakt berichten die Professorinnen Susanne Baer und Theresia Degener über „das schwierige Verhältnis von Amt und Mandat".

„Quotenfrau? Nein“, so Susanne Baer, seit Februar erste verpartnerte Richterin des Bundesverfassungsgerichts, „nicht die Quote, sondern ein Kampf ganz vieler“ habe sie in ihr neues Amt getragen. Beim Bundesverfassungsgericht erfahre sie Wertschätzung „auch und gerade als Person und Persönlichkeit, also auch: lesbisch und feministisch und mehr“. Ihr Wunsch zum Ende der zwölfjährigen Amtsperiode: Ihre Perspektiven mögen ganz fein sichtbar geworden sein, trotz richterlicher Zurückhaltung, die ja „mit richterlicher Unabhängigkeit gepaart“ sei.

Auch Theresia Degener, im Herbst 2010 in den Ausschuss zur UN-Behindertenrechtskonvention gewählt, betont, nun „keine Lobbyistin“ mehr zu sein, „zum Leidwesen der deutschen Behindertenorganisationen“. Allein der Kampf ihrer Eltern habe ihr, ohne Arme und Hände zur Welt gekommen, den Zugang zu „normaler“ Bildung ermöglicht. Die zweifache Mutter kämpft nun selbst für inklusive Bildung. Ihr Wunsch: einmal ihre „Fußabdrücke zu hinterlassen“.

Samstagvormittag besuche ich die AG „Vaterschaftsanfechtungen durch die Behörde“, wo Rechtsanwältin Barbara Wessel den neuen $\mathbb{S} 1600$ Absatz 1 Nr. 5 BGB beleuchtet. Traurige Praxis: Das Anfechtungsrecht der Behörde ist mittlerweile zu einem Instrument zur Abschiebung vor allem ausländischer Mütter geworden. Oft legt die Behörde deutschen Männern nach Anerkennung der Vaterschaft eines Kindes einer ausländischen Mutter und mitunter jahrelangem Zusammenleben mit beiden - die Durchführung eines Vaterschaftstests nahe. Dies vordergründig, um klare Verhältnisse zu schaffen, letztlich aber nur, um die Untreue der ausländischen Partnerin zu „beweisen“ und die bis dahin gelebte sozial-familiäre Beziehung zu zerstören und sich damit vor allem einen Anfechtungsgrund gemäß \ 1600 Absatz 3 BGB zu schaffen.

Unter den Foren (unter anderem „Rechtsideale und Lebenswirklichkeit im Familienrecht“; „Feministische Rechtstheorie“) entscheide ich mich für die „Unabhängigen Aufklärerinnen“. Die Rechtsanwältinnen Claudia Burgsmüller, Petra Ladenburger und Professorin Julia Zinsmeister schildern hier ihre Erfahrungen bei der Aufklärung von Missbrauchsverdachtsfällen an zwei Internatsschulen, die nach außen kaum unterschiedlicher wirken könnten: der Odenwaldschule (Konzept: „Familienleben“ zwischen Lehrer/innen und Schüler/innen) und dem von Jesuiten geleiteten Aloisiuskolleg in Bonn/Bad Godesberg.
„Wer gegen sexuelle Übergriffe aufmuckte, flog von der Schule“, laut Claudia Burgsmüller das Fazit ehemaliger Odenwaldschüler, die sich freiwillig an sie wandten und ausdrücklich nur als „Betroffene“, nicht als „Opfer“ bezeichnet werden wollten. Von den die Mehrzahl stellenden über 115 männlichen Opfern sexueller Übergriffe haben sich 56 selbst gemeldet, 59 weitere wurden von ihnen benannt; die weiblichen Opfer meldeten sich fast alle selbst. Entschädigungsleistungen an die Opfer selbst sind bislang nicht ausgezahlt worden, da die Odenwaldschule als Institution um ihr Überleben kämpft.

„Da kann man sowieso nichts machen“ - laut Petra Ladenburger Grundhaltung vieler Betroffener am Aloisiuskolleg. Julia Zinsmeister berichtet gar von „institutionalisiertem Missbrauch“, sexueller Natur und durch das Züchtigungsrecht. Die Täter, zumeist Patres, seien zielgerichtet gedeckt worden.

Beim Abschlussforum am Sonntag wird das Thema „Innere Sicherheit" dreimal sehr unterschiedlich behandelt: aus der Perspektive der Zeitzeugin von Alexandra Goy, seit den 1970er Jahren feministische Rechtsanwältin und Notarin in Berlin; Dominique Grisard von der Universität Basel referiert über die Geschlechtergeschichte des Linksterrorismus in der Schweiz und Julia Bernstein von der Universität Köln untersucht die Bedeutung des Essens für die innere Sicherheit von Migrantinnen.

\section{Resolutionen des Abschlussplenums - der 37. Feministische Juristinnentag fordert:}

Zur AG „Aktuelle Themen der Nebenklage“: Die Gleichstellung der Rechte der Nebenklage mit denen der Verteidigung, insbesondere hinsichtlich der Anwesenheitsrechte, um künftig die Aushebelung der Nebenklage durch Terminkollisionen zu verhindern; die Beteiligung der Nebenklage an verfahrensbeendenden Deals und Einstellungen; Streichung der Rechtsmittelbeschränkung der Nebenklage in der StPO. Die Frage, ob von Vergewaltigungen betroffenen Frauen nunmehr generell von Strafanzeigen abzuraten sei, beantwortete das Abschlussplenum nahezu einstimmig mit NEIN - dies wirke vielmehr an einer Verschleierung und Erhöhung der Dunkelziffer mit.

Zur AG „Arbeitsarrangements und Rückkehrrechte: Neue Impulse durch die Mutterschutz- und Elternzeitrichtlinie zur Vereinbarkeit von Familie und Beruf? ": Die fristgerechte Umsetzung der EU-Richtlinie 2010/18 zum Elternurlaub und hierbei insbesondere Schaffung von Möglichkeiten zur Durchsetzung von Arbeitsarrangements unabhängig von der Arbeitszeitreduzierung.

Zum Forum „Gender Pay Gap: Diskriminierung oder Entscheidung? “: Die Einführung eines Klagerechts für die Antidiskriminierungsstelle bei mittelbarer Diskriminierung sowie eines Verbandsklagerechts im Bereich der Entgeltdiskriminierung; ein Gesetz zur effektiven Durchsetzung der Entgeltgleichheit; die Änderung des HGB dahingehend, dass im Geschäftsbetrieb eines Unternehmens in anonymisierter Form die Entgeltstrukturen in Unternehmen für die verschiedenen Ebenen nach Frauen und Männern getrennt ausgewiesen werden müssen, sowie die Einführung eines allgemeinen, branchenübergreifenden Mindestlohns. 
Fazit: Frauenthemen sind zeitlos - trotz scheinbar unterschiedlicher Lebenswirklichkeiten. Damit es weitergehen kann: Sprecht die Älteren an.
Der 38. FJT wird im Mai 2012 in Bremen stattfinden. Die Inhalts- und die Organisationsgruppe suchen jeweils noch dringend Verstärkung!

\section{Der djb geht zu Hauptversammlungen: Ein Erfahrungsbericht}

\author{
Kivilcim Simsek \\ Mitarbeiterin des djb-Projekts „Aktionärinnen fordern Gleichbe- \\ rechtigung“; Rechtsanwältin, Berlin
}

Auch 2011 hat sich der djb auf die Fahne geschrieben, von den großen DAX, MDAX und TecDAX Unternehmen so viele wie möglich durch das Fragerecht dank Aktienbesitz bei den Hauptversammlungen auf das Thema Frauen in Führungspositionen anzusprechen. 75 Unternehmen wurden von vielen ehrenamtlichen Teilnehmerinnen und zwei Projektmitarbeiterinnen - eine davon ich - und in den unterschiedlichsten Städten Deutschlands besucht. In den wenigen Wochen lernte ich so die verschiedensten Unternehmen, Versammlungsorte und Bahnhöfe kennen.

Obwohl der Ablauf im Wesentlichen immer gleich war, stellte jede Hauptversammlung auch individuelle Anforderungen an mich als Rednerin. Die gefüllte Messe-Halle in Frankfurt oder die Olympiahalle in München forderten den Schneid, unbequeme Fragen vor 5.000 und mehr Menschen zu stellen. An anderen Tagen stellten Kleinaktionäre im Anschluss an die Redebeiträge unbequeme Fragen an mich. Lob und positive Resonanzen motivierten aber auch immer, das Thema voranzutreiben.

Der Besuch einer Hauptversammlung beginnt mit der Anmeldung der Fragen des djb, die sechs Tage zuvor per Post an die jeweiligen Unternehmen geschickt wurden. Daher waren alle Unternehmen, die wir besucht haben, bereits über unsere Teilnahme informiert. Zur Sicherheit reichten wir unsere Fragen regelmäßig am Versammlungstag noch einmal am Wortmeldetisch mit der Bitte um detaillierte Beantwortung ein.

Sodann begann jede Hauptversammlung mit einem Lagebericht des Aufsichtsratsvorsitzenden, der anschließend das Wort dem Vorstandsvorsitzenden übergab. Dieser berichtete von dem abgelaufenen Geschäftsjahr und über die Ziele des bevorstehenden Geschäftsjahrs. Teilweise kam es vor, dass der Aufsichtsratsvorsitzende oder der Vorstandsvorsitzende bereits dort das Thema „Frauen in Führungspositionen“ ansprachen. Allerdings gingen diese Bemerkungen meist nicht über das bereits im Geschäftsbericht Veröffentlichte hinaus. Vorwiegend teilten sie flüchtig mit, ob sie den Empfehlungen des Corporate Governance Kodexes entsprochen haben oder nicht. Unter großer Zustimmung im Publikum betonten manche auch kurz, dass sie gegen eine Frau- enquote seien oder behaupteten oberflächlich, dass es im Interesse des Unternehmens nur auf die Qualifikation, Erfahrungen und Kompetenzen der Kandidatinnen und Kandidaten ankäme.

Das Auditorium setzte sich neben den professionellen Aktionärsvertreterinnen und -vertretern meist aus älteren Aktionärinnen und Aktionären, Rentnerinnen und Rentnern zusammen. Neben dem Interesse am Unternehmen schenkten diese auch dem kostenfreien Mittagessen ihre Aufmerksamkeit und verließen den Saal pünktlich zur Buffeteröffnung. Ich kann nur raten, ob es Zufall war, dass unsere Redebeiträge meist zu dieser Mittagszeit stattfanden. Nach über 20 Versammlungen schienen viele der Aktionärinnen und Aktionäre, die ebenfalls häufig Versammlungen besuchten, schon fast klassisch nach Pawlow konditioniert bei der Nennung unserer Namen zu reagieren; des Öfteren standen Aktionärinnen und Aktionäre mit Redebeginn auf und verließen den Saal. Ebenfalls fielen gelegentlich abfällige Bemerkungen, auch von Aktionärinnen.

Dennoch bekamen wir nach unserem Wortbeitrag zumeist zustimmenden Beifall, und auch Szenenapplaus hat es gegeben. Auf der Hauptversammlung der ElringKlinger AG in Stuttgart bekamen wir allerdings gar keinen Beifall. Als zweite Teilnehmerin war ich die Einzige im Saal, die unserem Wortbeitrag applaudierte.

In der Regel wurden die Hauptversammlungen zu zweit besucht, meist waren es ein ehrenamtliches Mitglied des djb, unterstützt insbesondere bei der Protokollführung durch eine Projektmitarbeiterin.

Während die erste Teilnehmerin Fragen zu den Empfehlungen des Corporate Governance Kodexes stellte, waren die Fragen der zweiten Teilnehmerin auf konkrete Zahlen und Fakten hinsichtlich der Frauenbeteiligung im Unternehmen gerichtet. Sofern ich die Hauptversammlungen alleine besuchte, wurden beide Wortbeiträge zu einem zusammengefügt.

Bei den meisten Versammlungen wurden die Redebeiträge auf Leinwände und Fernseher übertragen. So wurde sichergestellt, dass nicht nur im Veranstaltungssaal selbst, sondern auch am Buffet und akustisch sogar auf den Toiletten den Wortbeiträgen gelauscht werden konnte. Es war nicht möglich uns nicht zu hören. Auch wenn manche den Saal verließen, weil wir in der Rednerliste weit hinten standen oder das Mittagessen rief, waren wir sicher, dass die Anwesenden das Thema hörten. 